\title{
Sensitivity and Specificity of HRT and OCT to Detect Diabetic Macular Edema
}

\author{
Subramaniam Suresh, Embong Zunaina*, Ismail Shatriah and Ab-Rahman Azriani
}

School of Medical Sciences, Universiti Sains Malaysia, 16150 Kubang Kerian, Kelantan, Malaysia

\begin{abstract}
Objectives: The aim of this study is to determine the sensitivity and specificity between leaking status using FFA and edema status using HRT III and OCT to detect macular edema, and also to determine the agreement of edema status seen between HRT III and OCT in diabetic macular edema (DME).

Methodology: Sixty seven patients with DME were selected for this study. They were subjected to HRT III and OCT to get edema values. Finally FFA examination was done to see the leaking area and served as the gold standard in this study. The results using HRT III and OCT were each compared with FFA, and the agreement of edema status between HRT III and OCT were analysed.

Results: Topographic edema map of HRT III has relatively better sensitivity and specificity $(78.1 \%$ and $76.6 \%$ respectively) compare with edema index of HRT III (74.2\% and $72.6 \%$ respectively) to detect DME. Topographic edema map of OCT has relatively better sensitivity and specificity $(68.7 \%$ and $85.0 \%$ respectively) compare with macular thickness value of OCT (48.6\% and $78.1 \%$ respectively) to detect DME. There is fair agreement $(r=0.252, p<0.001)$ between edema index of HRT III and macular thickness value of OCT, while other modules of HRT III and OCT has moderately good agreement $(r=0.455$ to $0.497, p<0.001)$ in detecting edema status in DME patients
\end{abstract}

Conclusion: Both topographic edema map of HRT III and OCT have relatively better sensitivity compared with other modules to detect DME. Good agreement exists between HRT III and OCT in detecting edema in DME patients.

Keywords: Diabetic macular edema; Fundus Fluorescein Angiography (FFA); Heidelberg Retinal Tomograph (HRT); Optical Coherence Tomography (OCT)

Abbreviation: DME: Diabetic Macular Edema; WESDR Wisconsin Epidemiologic Study of Diabetic Retinopathy; FFA: Fundus Fluorescein Angiography; HRT: Heidelberg Retinal Tomograph; OCT: Optical Coherence Tomography; ETDRS: Early Treatment Diabetic Retinopathy Study; HRA: Heidelberg Retina Angiography; SD: Standard Deviation

\section{Introduction}

Diabetic macular edema (DME) is an edema seen in the macular area as a result of diabetes mellitus complications. Chronic hyperglycemia itself initiates the process and leading to vascular abnormalities among diabetic patients. The main clinical features of DME are based on the location and severity of retinal thickening with presence of hard exudates (lipid deposits) [1]. DME was further classified into focal or diffuse, however there is no definite border to differentiate between these two terms. DME is divided into mild, moderate and severe type based on Diabetic Macular Edema Disease Severity Scale [2].

Clinically significant macular edema (CSME) was defined by the Early Treatment Diabetic Retinopathy Study (ETDRS) and demonstrated that eyes with CSME benefited from focal argon laser photocoagulation treatment when compared to untreated eyes in control. Treatment reduced the risk of moderate visual loss by $50 \%$, increased the chance of visual improvement, and was associated with only minor loses of visual field [3].

Wisconsin Epidemiologic Study of Diabetic Retinopathy (WESDR) is very well established and accepted worldwide. The latest report on WESDR XXIII which was published in 2009 highlighted that the 25year cumulative incidence of macular edema was $29 \%$ and $17 \%$ for CSME. The prevalence of diabetes in Malaysia is $11.6 \%$ with about 1.84 million of people were estimated suffering diabetes mellitus [4]. One of the earliest report published by Mafauzy using direct ophthalmoscope was found that about $23.5 \%$ of the studied population had background retinopathy and $5.3 \%$ had advanced diabetic eye disease $[5,6]$.

The standard tool for diagnosing diabetic macular edema is contact lens biomicroscopy. Fundus fluorescein angiography (FFA) has been used to evaluate vascular leakage qualitatively in assessing diabetic macular edema [7]. FFA can detect treatable leaking points and evaluation of ischemic area prior to treatment. FFA is an invasive intervention which is not appropriate for routine screening test.

Heidelberg Retinal Tomograph (HRT) is a Confocal Scanning Laser Ophthalmoscope which is a non-invasive method with laser scanning system. HRT III has 3 modules, first topographic edema mapping which is drafted by software based on vitreous interface elevation and it gives an image similar to those used in geographic land mapping. Second is the macular edema index thickness mapping which is constructed based on z-profile image pixel (each pixel has about 10 $\mu \mathrm{m})$ [8]. Finally, edema index is a numeric value determining edema present or not [9]. Ang et al. reported that HRT has the capacity to detect small increases in macular volume that may not be detected by clinical evaluation and examination [10].

*Corresponding author: Embong Zunaina, Department of Ophthalmology, Schoo of Medical Sciences, Universiti Sains Malaysia, 16150 Kubang Kerian, Kelantan, Malaysia, Tel: 609767 6361; Fax: 609765 3370; E-mail: zunaina@kb.usm.my

Received August 12, 2011; Accepted September 20, 2012; Published September 25, 2012

Citation: Suresh S, Zunaina E, Shatriah I, Azriani AR (2012) Sensitivity and Specificity of HRT and OCT to Detect Diabetic Macular Edema. J Diabetes Metab S3:009. doi:10.4172/2155-6156.S3-009

Copyright: ( 2012 Suresh S, et al. This is an open-access article distributed unde the terms of the Creative Commons Attribution License, which permits unrestricted use, distribution, and reproduction in any medium, provided the original author and source are credited. 
New investigation tool Optical Coherence Tomography (OCT) was invented to give better quantitative estimation of retinal thickness at multiple points within macular region by constructing a retinal thickness map. Lang published data regarding the sensitivity of OCT in detecting the smaller retinal thickness changes. The result shows that OCT has better sensitiveness in detecting smaller changes in retinal thickness compare to fundus biomicroscopy [11].

The aim of this study is to determine the sensitivity and specificity between leaking status using FFA and edema status using HRT III and OCT to detect DME. We also want to determine the agreement of edema status seen between HRT III and OCT in DME.

\section{Methods}

\section{Participants}

We recruited diabetic patients with DME and excluded those with other possible causes of macular edema and there is no contraindication to undergo contrast study. A total of $67 \mathrm{DME}$ patients presented to Eye Clinic, Hospital Universiti Sains Malaysia, Kelantan, Malaysia between December 2009 to July 2011 were included into this study.

\section{Classification of DME}

The classification of DME is based on Diabetic Macular Edema Disease Severity Scales [2] with modification (Table 1). An ETDRS grid (nine sectors of macular area) was used as a guideline for the specific distance from the fovea (Figure 1). In this study, DME was grouped into mild, moderate and severe with FFA guideline (Figure 2). Any edema seen within $1 \mathrm{~mm}$ circle at central macular was termed as severe type DME. Edema seen between $1 \mathrm{~mm}$ and $3 \mathrm{~mm}$ circles was termed as moderate type DME. Edema seen between $3 \mathrm{~mm}$ and $6 \mathrm{~mm}$ circles was considered as mild form of DME. In this study, three modalities (FFA, HRT III and OCT) were used to detect macular edema and FFA was considered as a gold standard.

\section{Definition of term}

- Leaking Status of FFA

a) Positive edema leaking: hyperfluorescency area seen in the macular area after fluorescein dye injected intravenously.

b) Negative edema leaking: no hyperfluorescency seen in the macular area after fluorescein dye injected intravenously.

- Topographic Edema Map of HRT III

a) Positive edema topographic: the red and above color coding area from signal width map.

b) Negative edema topographic: the orange and below color coding area from signal width map.

- Edema Index of HRT III

a) Positive edema index: above the cut off macular edema index value $(\mathrm{e}>1.835)$ [9]. b) Negative edema index: below the cut off macular edema index value $(\mathrm{e} \leq 1.835)$ [9]

- Topographic Edema Map of OCT

a) Positive edema topographic: increase in the retinal mapping color coding.

b) Negative edema topographic: no increase in the retinal mapping color coding.

- Macular Thickness Value of OCT

a) Positive edema thickness: increase in the retinal thickness above the reference value based on location [12] (Table 2).

b) Negative edema thickness: decrease in the retinal thickness equal and below the reference value based on location [12] (Table 2).

\section{Study procedure}

Patient's demographic data and eye examination findings were documented. Topical Mydfrin 2.5\% (phenylephrine hydrochloride 2.5\%) and topical Mydriacyl 1.0\% (tropicamide 1.0\%) were instilled every 5 minutes for 15 minutes until pupil is mid-dilated. Fundus photo was taken for documentation before proceed with non-invasive procedures first (HRT III and OCT) and then followed with invasive procedure (FFA examinations).

HRT III (Heidelberg Engineering, Heidelberg, Germany) was used for evaluation of topographic edema map and edema index. HRT III examination was done on patient's selected eye. The patient sits in front of HRT III monitor and focused at given target. Patient's eye kept focused until get a good picture of his/her posterior pole with fovea as central locus. The captured image at this point was divided into nine sectors of macular area. However, HRT III area of focusing is $4.4 \times 4.4 \mathrm{~mm}$. Data with image printed for review. The evaluation of the edematous status base on topographic edema map and edema index evaluations were done by an investigator $\mathrm{A}$.

OCT (Heidelberg Engineering, Heidelberg, Germany) was used for evaluation of topographic edema map and macular thickness value. OCT examination was done on patient's selected eye. The patient sits in front of OCT monitor and focused at given target. Patient's eye kept focused until get a good picture of his/her posterior pole with fovea as central locus. The captured image at this point was divided into nine sectors of macular area (ETDRS grid). Data with image printed for review. The evaluations of the edematous status, both topographic edema map and macular thickness value were done by an investigator B.

At last, invasive FFA contrast study was done. Heidelberg Retina Angiography (HRA) (Heidelberg Engineering, Heidelberg, Germany) was used for evaluation of leaking edema status. Intravenous line set up and patency checked. Intravenous bolus of $1.5 \mathrm{ml}$ of sodium fluorescein $10 \%$ dye injected. As soon as the dye was injected, the image was recorded simultaneously using Heidelberg Retina Angiograph.

\begin{tabular}{lll}
\hline $\begin{array}{l}\text { Severity of diabetic macular } \\
\text { edema }\end{array}$ & Diabetic Macular Edema Disease Severity Scales [2] & $\begin{array}{l}\text { Modified Diabetic Macular Edema Disease Severity } \\
\text { Scales }\end{array}$ \\
\hline Mild & $\begin{array}{l}\text { some retinal thickening or hard exudates in posterior pole but distant from the } \\
\text { center of the macula }\end{array}$ & $\begin{array}{l}\text { edema seen between 3mm and 6mm circles at } \\
\text { central macular }\end{array}$ \\
\hline Moderate & $\begin{array}{l}\text { retinal thickening or hard exudates approaching the center of the macula but not } \\
\text { involving the center }\end{array}$ & $\begin{array}{l}\text { edema seen between } 1 \mathrm{~mm} \text { and 3mm circles at } \\
\text { central macular }\end{array}$ \\
\hline Severe & retinal thickening or hard exudates involving the center of the macula & edema seen within 1mm circle at central macular \\
\hline
\end{tabular}

Table 1: Classification of diabetic macular edema. 
Citation: Suresh S, Zunaina E, Shatriah I, Azriani AR (2012) Sensitivity and Specificity of HRT and OCT to Detect Diabetic Macular Edema. J Diabetes Metab S3:009. doi:10.4172/2155-6156.S3-009

Page 3 of 6

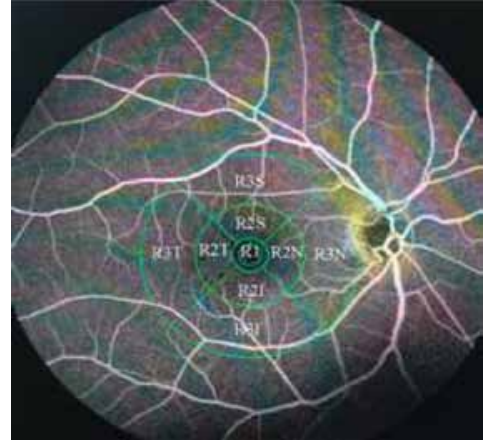

Figure 1: The ETDRS grid (nine sectors of macular area) on captured image of FFA from Heidelberg Retina Angiography (HRA) (R1: $1 \mathrm{~mm}$ diameter; R2: $3 \mathrm{~mm}$ diameter; R3: $6 \mathrm{~mm}$ diameter from the centre of macula; $\mathrm{T}$ : temporal; $\mathrm{N}$ : Nasal; S: Superior; I: linferior)
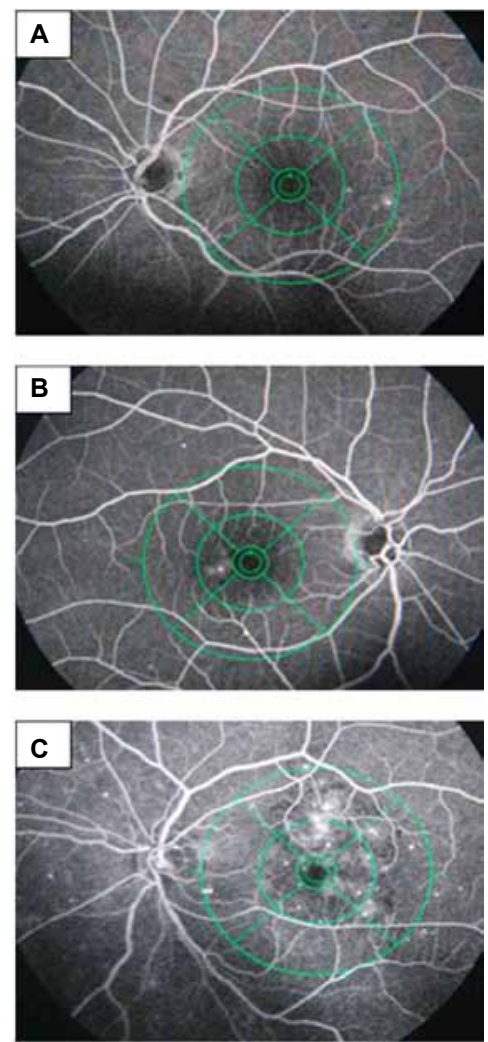

Figure 2: Diabetic macular edema classification with FFA guideline. A: Mild DME; B: Moderate DME; C: Severe DME.

Those patients who gave history of allergy, steroid coverage given based on standard regime which is $50 \mathrm{mg}$ at 13 hours, 7 hours and 1 hour before procedure. This procedure is done by principal investigator. The evaluation of the leaking status of FFA at nine sectors of macular area (ETDRS grid) with fovea as central point is done by an investigator C.

\section{Ethical approval}

The study was approved by the Research and Ethical Committee, School of Medical Sciences, Universiti Sains Malaysia (Ref: USMKK/ PPP/JEPeM [221.4.(1.5)]).

\section{Statistical analysis}

All the statistical method analysis was done with Statistical Package for Social Sciences (SPSS Inc.) software, version 18.0. The validity of HRT III and OCT procedure is tested for its ability to distinguish between edema present and not present among DME patients by comparing to the gold standard FFA procedure. Validity has two components which are sensitivity and specificity. The sensitivity is the ability of HRT III or OCT to identify edema correctly those who have detected edema by FFA. The specificity is the ability of HRT III or OCT to identify those who do not have edema by FFA. Crosstabs Statistic was used to derive the kappa value to determine the agreement of macular edema status seen between HRT III and OCT in diabetic patient with DME ( $\mathrm{p}$-value $<0.05$, significant). The $\kappa$ statistic is interpreted as the chance-corrected proportional agreement between the two groups. The $\kappa$ value has a maximum of 1.00 when agreement is perfect, and $\kappa=0$ indicates no agreement better than chance.

\section{Results}

A total of 67 patients that fulfilled the inclusion and exclusion criteria were recruited for this study. The mean age was $55.67, \mathrm{SD} \pm$ 6.1 years (range: $38-67$ years) with male participants were 38 patients (56.7\%) and female participants were 29 patients (43.3\%). There were 11 patients (16.4\%) with mild DME, 39 patients $(58.2 \%)$ with moderate DME and 17 patients (25.4\%) with severe DME.

\section{Sensitivity and specificity of HRT III to detect macular edema}

Topographic edema map of HRT III to detect macular edema among DME, moderate DME has better sensitivity compare to other severity type of DME to detect edema status (sensitivity $81.9 \%$ ) and mild DME showed least sensitivity value, $60.7 \%$. The specificity is better among mild type DME, 84.5\% and least among severe DME, 64.7\% (Table 3). Edema index evaluation for macular edema using HRT III among DME, severe DME has better sensitivity compared to other severity type of DME to detect edema status (sensitivity 76.5\%) and mild DME showed least sensitivity value, $67.9 \%$. The specificity is better among severe type DME, $82.4 \%$ and least among moderate DME, 66.9\% (Table 3).

\section{Sensitivity and specificity of OCT to detect macular edema}

Topographic edema map of OCT to detect macular edema among DME, severe DME has better sensitivity compared to other severity

\begin{tabular}{ll}
\hline Subfield & $\begin{array}{l}\text { Mean retinal Thickness }(\mu \mathrm{m}) \\
\text { Mean } \pm \text { SD }\end{array}$ \\
\hline Central subfield & $270.2 \pm 22.5$ \\
Superior inner macula & $336.0 \pm 20.6$ \\
Nasal inner macula & $335.0 \pm 19.3$ \\
Inferior inner macula & $334.9 \pm 16.7$ \\
Temporal inner macula & $322.6 \pm 16.5$ \\
Superior outer macula & $329.6 \pm 16.4$ \\
Nasal outer macula & $339.5 \pm 16.9$ \\
Inferior outer macula & $325.4 \pm 16.6$ \\
Temporal outer macula & $320.1 \pm 15.4$
\end{tabular}

Table 2: Reference value of mean retinal thickness in the nine ETDRS subfields [12]. 
Citation: Suresh S, Zunaina E, Shatriah I, Azriani AR (2012) Sensitivity and Specificity of HRT and OCT to Detect Diabetic Macular Edema. J Diabetes Metab S3:009. doi:10.4172/2155-6156.S3-009

Page 4 of 6

type of DME to detect edema status (sensitivity 74.8\%) and mild DME showed least sensitivity value, $50.0 \%$. The specificity is better among mild type DME, $97.2 \%$ and least among severe DME, 79.4\% (Table 4). In macular thickness value evaluation for macular edema using OCT among DME, severe DME has better sensitivity compared to other severity type of DME to detect edema status (sensitivity $58.8 \%$ ) and mild DME showed least sensitivity value, $17.9 \%$. The specificity is better among mild type DME, $93.0 \%$ and least among moderate DME, $71.6 \%$ (Table 4).

\section{Agreement between HRT III and OCT to detect macular edema}

There was moderately good agreement existed between topographic edema map and edema index of HRT III ( $\kappa$ value 0.455 ; $\mathrm{p}$ value $<0.001)$. There was also moderately good agreement existed between topographic edema map and macular thickness value of OCT ( $\kappa$ value 0.497; p value $<0.001$ ). Evaluation between HRT III and OCT showed moderately good agreement of topographic edema map existed between HRT III and OCT ( $\kappa$ value 0.485 ; $p$ value $<0.001$ ) and fairly

\begin{tabular}{llll}
\hline Type of DME & \multicolumn{2}{l}{ Topographic Edema Map HRT III } & \multicolumn{2}{c}{ Edema Index HRT III } \\
& Sensitivity (\%) & Specificity (\%) & Sensitivity (\%) \\
\hline Mild & 60.7 & 84.5 & 67.9 \\
Moderate & 81.9 & 80.5 & 73.6 \\
Severe & 76.5 & 64.7 & 76.5 \\
All DME & 78.1 & 76.6 & 74.2 \\
\hline
\end{tabular}

Table 3: Sensitivity and specificity of HRT III modules to detect macular edema.

\begin{tabular}{llll}
\hline Type of DME & \multicolumn{2}{l}{ Topographic Edema Map OCT } & \multicolumn{2}{c}{ Macular Thickness Value OCT } \\
& Sensitivity (\%) & Specificity (\%) & \multicolumn{2}{c}{ Sensitivity (\%) } \\
\hline Mild & 50.0 & 97.2 & 17.9 \\
Moderate & 67.6 & 81.1 & 46.7 \\
Severe & 74.8 & 79.4 & 58.8 \\
All DME & 68.7 & 85.0 & 48.6
\end{tabular}

Table 4: Sensitivity and specificity of OCT modules to detect macular edema.

\begin{tabular}{|c|c|c|}
\hline & $\begin{array}{l}\text { Kappa value } \\
\text { (p-value) }\end{array}$ & Agreement \\
\hline \multicolumn{3}{|c|}{ Topographic Edema Map (HRT III) vs Edema Index (HRT III) } \\
\hline Mild DME & $0.427(<0.001)$ & moderate \\
\hline Moderate DME & $0.428(<0.001)$ & moderate \\
\hline Severe DME & $0.424(<0.001)$ & moderate \\
\hline All DME & $0.455(<0.001)$ & moderate \\
\hline \multicolumn{3}{|c|}{ Topographic Edema Map (OCT) vs Macular Thickness Value (OCT) } \\
\hline Mild DME & $0.473(<0.001)$ & moderate \\
\hline Moderate DME & $0.437(<0.001)$ & moderate \\
\hline Severe DME & $0.489(<0.001)$ & moderate \\
\hline All DME & $0.497(<0.001)$ & moderate \\
\hline \multicolumn{3}{|c|}{ Topographic Edema Map (HRT III) vs Topographic Edema Map (OCT) } \\
\hline Mild DME & $0.542(<0.001)$ & Moderate \\
\hline Moderate DME & $0.427(<0.001)$ & Moderate \\
\hline Severe DME & $0.441(<0.001)$ & Moderate \\
\hline All DME & $0.485(<0.001)$ & Moderate \\
\hline \multicolumn{3}{|c|}{ Edema Index (HRT III) vs Macular Thickness Value (OCT) } \\
\hline Mild DME & $0.099(0.207)$ & Slight \\
\hline Moderate DME & $0.199(<0.001)$ & Slight \\
\hline Severe DME & $0.319(<0.001)$ & Fair \\
\hline All DME & $0.252(<0.001)$ & Fair \\
\hline
\end{tabular}

$p$ value $<0.05$ is significant

McNemar statistical test

Table 5: Agreement of edema status between HRT III and OCT to detect macular edema 
good agreement existed between edema index of HRT III and macular thickness value of OCT ( $\kappa$ value 0.252 ; $\mathrm{p}$ value $<0.001$ ) (Table 5).

\section{Discussion}

The mean age from this study fall at 55.67 ( $S D \pm 6.1$ ) years (range: 38-67 years). These findings are relevant to previous studies done on diabetic retinopathy screening where most of participants are middle aged group with mean age was $58.1(\mathrm{SD} \pm 14.4)$ years and 54 years in Singapore and Malaysia respectively [13-17].

In our study, two modalities of HRT III (topographic edema map and edema index) were compared with FFA leaking status and the sensitivity value was above $70 \%$. Guan et al. compared HRT and Retinal Thickness Analyzer in detecting DME, where the outcome showed that HRT has sensitivity $92 \%$ and specificity $68 \%$ to detect DME [18]. We found that topographic edema map of HRT III has better sensitivity and specificity $(78.1 \%$ and $76.6 \%$ respectively) compared to edema index of HRT III (74.2\% sensitivity and $72.6 \%$ specificity). Further on assessing based on severity, moderate DME has better sensitivity and specificity (topographic edema map: $81.9 \%$ and $80.5 \%$ ). As par with the classification, moderate DME occupied relatively wider area (between $1 \mathrm{~mm}$ and $3 \mathrm{~mm}$ ) and this area is rich with vessels. HRT III topographic edema map is a better choice for those who are suffering from moderate DME and unable to do invasive procedure to identify leaking area accurately. Multiple colors coding as a reference in topographic edema map play an important role in deciding area with edema and no edema.

Zambarakji et al. published a paper on volumetric analysis; the outcome shows that volumetric analysis has sensitivity $81.82 \%$ in detecting DME with edema index cut off point 1.8 [19]. In this study, we used edema index cut off point 1.835 [8] and the sensitivity and specificity of edema index to detect edema in DME patient was $74.2 \%$ and $72.6 \%$ respectively. The sensitivity and specificity discrepancies were mainly due to the difference in setting the edema index cut off point.

SCORE system (System for Classification and Ordering of Retinal Edema) was introduced using data obtained from HRT (developed using subjective assessment of the color map and the reflectivity image) and the outcome of this SCORE system has sensitivity and specificity $67 \%$ and $99 \%$ respectively [20]. In our study, we found that topographic edema map in HRT III has sensitivity and specificity of $78.1 \%$ and 76.6\% respectively to detect edema in DME patients.

In our study, we assessed the agreement between topographic edema map and edema index of HRT III, we found that there was good agreement existing between topographic edema map and edema index of HRT III $(\mathrm{r}=0.455, \mathrm{p}<0.001)$.

OCT was tested for macular thickness value by comparing to normal value published [12]. With this value cut off points, it is possible to plot edema area according to ETDRS grid (nine sectors) from thickness value obtained from OCT.

Introduction of OCT triggered various studies to determine and establish its uses into our daily clinical practice. In this study, we found that the topographic edema map and macular thickness evaluation has sensitivity $68.7 \%$ and $48.6 \%$ respectively. One of the earliest OCT study by Sanchez-Tocino et al., OCT has sensitivity of $93 \%$ to detect edema at central macular area $1 \mathrm{~mm}$ (fovea) with cut off foveal thickness $180 \mu \mathrm{m}$ (reference value) [21]. In another study by Goebel and KretzchmarGross, it was stated that sensitivity to detect edema among CSME was $89 \%$ and specificity was $96 \%$ [22]. Kozak et al. stated in their publication that overall evaluation of OCT has sensitivity about $96.1 \%$
[23]. The discrepancies between our studies with others were widely due to no normal data available to set as reference value for Malaysian population. Apart this, area of interest in our study is $6 \mathrm{~mm}$ diameter with fovea centre.

Browning et al. published a paper regarding measurements and analysis OCT done on the central macula. Diagnosing DME involving macula needs high resolution images because the retinal thickness varies and fovea is the thinnest part of posterior pole [24].

Topographic edema map from OCT and HRT III was used to analyze based on severity of DME. We found that, mild DME interestingly shows better agreement with $\kappa$ value $0.542(p<0.001)$ compared to moderate and severe type. Minimal and early DME changes can be seen through topographic edema map of OCT. Thus this module can be used for fast screening of diabetic retinopathy or DME.

Detecting edema among diabetic patients by topographic edema map (HRT III and OCT) shows moderately good agreement ( $\kappa$ value $0.485 ; \mathrm{p}<0.001)$. However topographic edema map and macular thickness value of OCT evaluation has better agreement with $\kappa$ value $0.497(p<0.001)$. Macular edema can be quantitatively mapped by OCT (macular thickness value) and HRT III (edema index) and data from published paper shows both significantly $(\mathrm{p}<0.05)$ correlate to each other [25]. In our study, we assessed the agreement between HRT III (edema index) and OCT (macular thickness values), the outcome shows there is fairly good agreement exist ( $\kappa$ value $0.252 ; \mathrm{p}<0.001$ ).

\section{Limitation}

Area of interest in this study is $6 \mathrm{~mm}$ diameter circle with fovea center, however HRT III area of focusing is $4.4 \times 4.4 \mathrm{~mm}$. There is missing area of interest in HRT III, however outside $3 \mathrm{~mm}$ was considered mild type DME and the uncovered area was ignored. In future if HRT III plans to improve their software, hope this can put as consideration for better coverage. Apart this, we also do not have normal data available for macular thickness value of OCT to set as reference value for Malaysian population that resulted in low sensitivity value.

\section{Conclusion}

Both topographic edema map of HRT III and OCT have relatively better sensitivity compare with other modules to detect DME. There is fair agreement between edema index of HRT III and macular thickness value of OCT, while other modules of HRT III and OCT have moderately good agreement in detecting edema status in DME patients.

\section{Acknowledgements}

This study was supported by a research grant (1001/PPSP/812064) from Universiti Sains Malaysia.

\section{References}

1. Funatsu H, Yamashita $H(2005)$ Pathogenesis of diabetic retinopathy and diabetic macular edema and enzyme inhibition. Current Enzyme Inhibition 1 329-337.

2. Wilkinson CP, Ferris FL 3rd, Klein RE, Lee PP, Agardh CD, et al. (2003) Proposed international clinical diabetic retinopathy and diabetic macular edema disease severity scales. Ophthalmology 110: 1677-1682.

3. (1995) Focal photocoagulation treatment of diabetic macular edema Relationship of treatment effect to fluorescein angiographic and other retina characteristics at baseline: ETDRS report no. 19. Arch Ophthalmol 113: 11441155.

4. Shaw JE, Sicree RA, Zimmet PZ (2010) Global estimates of the prevalence of diabetes for 2010 and 2030. Diabetes Res Clin Pract 87: 4-14. 
Citation: Suresh S, Zunaina E, Shatriah I, Azriani AR (2012) Sensitivity and Specificity of HRT and OCT to Detect Diabetic Macular Edema. J Diabetes Metab S3:009. doi:10.4172/2155-6156.S3-009

5. Mafauzy M (2005) Diabetes control and complications in private primary healthcare in Malaysia. Med J Malaysia 60: 212-217.

6. Mafauzy M (2006) Diabetes control and complications in public hospitals in Malaysia. Med J Malaysia 61: 477-483.

7. Virgili G, Menchini F, Dimastrogiovanni AF, Rapizzi E, Menchini U, et al. (2007) Optical Coherence Tomography versus stereoscopic fundus photography or biomicroscopy for diagnosing diabetic macular edema: A systematic review. Invest Ophthalmol Vis Sci 48: 4963-4973.

8. Hudson C, Flanagan JG, Turner GS, McLeod D (1998) Scanning laser tomography $\mathrm{Z}$ profile signal width as an objective index of macular retinal thickening. Br J Ophthalmol 82: 121-130.

9. Subrayan V, Chuah A, Peyman M, Pei LV, lqbal T (2010) Scanning laser edema index. Ophthalmology 117: 2233.e1-2233.e2.

10. Ang A, Tong L, Vernon SA (2000) Improvement of reproducibility of macular volume measurements using the Heidelberg Retinal Tomograph. $\mathrm{Br} \mathrm{J}$ Ophthalmol 84: 1194-1197.

11. Lang GE (2007) Optical coherence tomography findings in diabetic retinopathy. Dev Ophthalmol 39: 31-47.

12. Grover S, Murthy RK, Brar VS, Chalam KV (2009) Normative data for macular thickness by high-definition spectral-domain optical coherence tomography (spectralis). Am J Ophthalmol 148: 266-271.

13. Merriman A, Ross I (1985) Findings among 100 type 2 diabetics in a clinic in Penang, Malaysia, 1983-84. Ann Acad Med Singapore 14: 277-285.

14. Lee WR, Lim HS, Thai AC, Chew WL, Emmanuel S, et al. (2001) A window on the current status of diabetes mellitus in Singapore--the Diabcare-Singapore 1998 study. Singapore Med J 42: 501-507.

15. Massin P, Aubert JP, Eschwege E, Erginay A, Bourovitch JC, et al. (2005) Evaluation of a screening program for diabetic retinopathy in a primary care setting Dodia (Dépistage ophtalmologique du diabète) study. Diabetes Metab 31: 153-162.
16. Wong TY, Cheung N, Tay WT, Wang JJ, Aung T, et al. (2008) Prevalence and risk factors for diabetic retinopathy: The Singapore Malay Eye Study. Ophthalmology 115: 1869-1875.

17. Goh PP, Omar MA, Yusoff AF (2010) Diabetic eye screening in Malaysia: findings from the National Health and Morbidity Survey 2006. Singapore Med J 51: 631-634

18. Guan K, Hudson C, Flanagan JG (2004) Comparison of Heidelberg Retina Tomograph II and Retinal Thickness Analyzer in the assessment of diabetic macular edema. Invest Ophthalmol Vis Sci 45: 610-616.

19. Zambarakji HJ, Butler TK, Vernon SA (1999) Assessment of the Heidelberg Retina Tomograph in the detection of sight-threatening diabetic maculopathy. Eye (Lond) 13: 136-144.

20. Tong L, Ang A, Vernon SA, Zambarakji HJ, Bhan A, et al. (2001) Sensitivity and specificity of a new scoring system for diabetic macular oedema detection using a confocal laser imaging system. Br J Ophthalmol 85: 34-39.

21. Sánchez-Tocino $H$, Alvarez-Vidal A, Maldonado MJ, Moreno-Montañés J García-Layana A (2002) Retinal thickness study with optical coherence tomography in patients with diabetes. Invest Ophthalmol Vis Sci 43: 1588-1594.

22. Goebel W, Kretzchmar-Gross T (2002) Retinal thickness in diabetic retinopathy A study using optical coherence tomography (OCT). Retina 22: 759-767.

23. Kozak I, Morrison VL, Clark TM, Bartsch DU, Lee BR, et al. (2008) Discrepancy between fluorescein angiography and optical coherence tomography in detection of macular disease. Retina 28: 538-544.

24. Browning DJ, Glassman AR, Aiello LP, Bressler NM, Bressler SB, et al. (2008) Optical coherence tomography measurements and analysis methods in optical coherence tomography studies of diabetic macular edema. Ophthalmology 115: $1366-1371$

25. Degenring RF, Aschmoneit I, Kamppeter B, Budde WM, Jonas JB (2004) Optical coherence tomography and confocal scanning laser tomography for assessment of macular edema. Am J Ophthalmol 138: 354-361.
This article was originally published in a special issue, Diabetic Retinopathy handled by Editor(s). Dr. Caroline J Magri, Mater Dei Hospital, Malta 